\title{
COMPARISON THEOREMS FOR FOURTH ORDER DIFFERENTIAL EQUATIONS
}

\author{
GARRET J. ETGEN \\ Department of Mathematics \\ University of Houston - University Park \\ Houston, Texas 77004 \\ WILLIE E. TAYLOR, JR. \\ Department of Mathematics \\ Texas Southern University \\ Houston, Texas 77004 \\ (Received April 23, 1984 and in revised form August 1, 1985)
}

ABSTRACT. This paper establishes an apparently overlooked relationship between the p'air of fourth order linear equations $y^{i v}-p(x) y=0$ and $y^{i v}+p(x) y=0$, where $p$ is a positive, continuous function defined on $[0, \infty)$. It is shown that if all solutions of the first equation are nonoscillatory, then all solutions of the second equation must be nonoscillatory as well. An oscillation criterion for these equations is also given.

KEY WORDS AND PHRASES. Oscillatory solutions; nonoscillatory solutions; fourth order linear differential equations.

1980 MATHEMATICS SUBJECT CLASSIFICATION CODE. $34 \mathrm{C} 10$

1. INTRODUCTION.

This note is concerned with the pair of fourth order linear differential equations

$$
\begin{aligned}
& y^{i v}-p(x) y=0 \\
& y^{i v}+p(x) y=0
\end{aligned}
$$

where $p=p(x)$ is a positive, continuous function on $[0, \infty)$. The objective is to relate the oscillatory character of (1.1) with that of (1.2).

Recall that a linear differential equation is said to be oscillatory on an interval I if it has a nontrivial solution $y=y(x)$ which has infinitely many zeros on I. Also, such a solution $y$ is called oscillatory. On the other hand, if no nontrivial solution of the equation is oscillatory, that is if all nontrivial solutions are nonoscillatory, then the equation is said to be nonoscillatory on I. Hereafter, the term "solution" shall be interpreted to mean "nontrivial solution".

This study is motivated by several recent results concerning pairs of equations of the form

$$
\begin{aligned}
& z^{(k)}-p(x) z=0 \\
& z^{(k)}+p(x) z=0
\end{aligned}
$$


Sce, for example, D. L. Lovelady [1] and W. E. Taylor, Jr., [2]. When the order, k, of the equations is odd, each equation is the adjoint of the other and it is natural that their oscillatory character should be related. In particular, (1.4) is oscillatory if and only if (1.3) is, when $k$ is odd. In contrast, (1.1) and (1.2) are each self-adjoint and the current literature suggests that the two equations are unrelated with respect to oscillatory behavior. Indeed, the two equations have always been studied separately. See the works of W. Leighton and Z. Nehari [3], M. Keener [4], and S. Ahmad [5] for detailed investigations of these equations. The fact that either all the solutions of (1.2) are oscillatory or they are all nonoscillatory, while (1.1) always has nonoscillatory solutions, provides a demonstration of the apparent independence of the two equations in terms of oscillation. However, contrary to this evidence, we shall show that there is a relationship between (1.1) and (1.2) that involves the oscillatory behavior of the solutions.

\section{MALN RESULTS.}

Our work requires the following general lemma. A proof of this result appears in the paper [6] by G. D. Jones.

LEMMA. If the function $f=f(x)$ is $(k+1)$-times differentiable and satisfies $f^{(i)}(x) \geq 0, \quad i=0,1, \ldots, k$, and $f^{(k+1)}(x)<0$ on an interval $[a, \infty)$, then

$$
\frac{f(x)}{f^{(i)}(x)} \geq \frac{(k-i) !}{k !}(x-a)^{i}, \quad 1 \leq i \leq k-1,
$$

on $[a, \infty)$.

Both Ahmad [5] and Keener [4] have shown that (1.1) always has a pair of solutions $y$ and $w$ satisfying

$$
y(x)>0, y^{\prime}(x)>0, y^{\prime \prime}(x)>0, y^{\prime \prime \prime}(x)>0
$$

on some half-line $[b, \infty), b \geq 0$, and

$$
w(x)>0, w^{\prime}(x)<0, \quad w^{\prime \prime}(x)>0, w^{\prime \prime}(x)<0
$$

on $[0, \infty)$. Solutions of (1.1) which satisfy (2.1) are said to be strongly increasing

on $[b, \infty)$, while solutions satisfying (2.2) are termed strongly decreasing. Ahmad proved that (1.1) is oscillatory if and only if all nonoscillatory, eventually positive solutions satisfy either (2.1) or (2.2). This fact serves as the basis for our main theorem.

THEOREM 1. If (1.1). is nonoscillatory, then (1.2) is nonoscillatory.

PROOF. Assume that (1.1) is nonoscillatory. It follows from the remarks above that (1.1) has an eventually positive solution which fails to satisfy either (2.1) or $(2.2)$. It is easy to show that such a solution, say $y=y(x)$, must satisfy

$$
y(x)>0, y^{\prime}(x)>0, y^{\prime \prime}(x)>0, y^{\prime \prime \prime}(x)<0
$$

on $[a, \infty)$ for some $a \geq 0$. Let $z=y^{\prime}(x)$. Then $z$ is a solution of the third order equation

$$
z^{\prime \prime}-\frac{p(x) y(x)}{y^{\prime}(x)} z=0
$$

and $z$ satisfies $z(x)>0, z^{\prime}(x)>0, z^{\prime \prime}(x)<0$ on $[a, \infty)$. From this we can conclude that $(2.3)$ is $(1,2)-d i s c o n j u g a t e$ on $[a, \infty)$ and hence nonoscillatory (see [2]). 
Using the Lemma, we have

$$
\frac{y(x)}{y^{\prime}(x)} \geq \frac{x-a}{2}
$$

Thus, by a wel1-known comparison theorem (see, for example, Nehari [7]), the third order equation

$$
z^{\prime \prime}-\frac{(x-a)}{2} p(x) z=0
$$

is also nonosrillatory and $(1,2)$-disconjugate on $[a, \infty)$. Therefore, (2.4) has a solution $u=u(x)$ satisfying $u(x)>0, u^{\prime}(x)>0, u^{\prime \prime}(x)<0$ on $[a, \infty)$. It follows from these incqualities that $u^{\prime \prime}(x) \rightarrow 0$ and $u^{\prime}(x) \rightarrow \beta \geq 0$ as $x \rightarrow \infty$. Substituting u into (2.4) yields

$$
u^{\prime \prime \prime}(x)=\frac{(x-a) p(x)}{2} u(x)
$$

We integrate this equation from $x$ to $s$ to obtain

$$
u^{\prime \prime}(s)-u^{\prime \prime}(x)=\frac{1}{2} \int_{x}^{s}(t-a) p(t) u(t) d t, \quad x \geq a .
$$

Now, letting $s \rightarrow \infty$ and using the fact that $u^{\prime \prime}(s) \rightarrow 0$, we have

$$
-u^{\prime \prime}(x)=\frac{1}{2} \int_{x}^{\infty}(t-a) p(t) u(t) d t
$$

Integrate again from $x$ to $s$, using integration by parts. The result is

$$
\begin{aligned}
u^{\prime}(x)-u^{\prime}(s) & =\frac{1}{2} \int_{x}^{s}\left[\int_{t}^{\infty}(r-a) p(r) u(r) d r\right] d t \\
& =\frac{1}{2}(s-x) \int_{s}^{\infty}(t-a) p(t) u(t) d t+\frac{1}{2} \int_{x}^{s}(t-x)(t-a) p(t) u(t) d t
\end{aligned}
$$

or

$$
u^{\prime}(x)=u^{\prime}(s)+\frac{1}{2}(s-x) \int_{s}^{\infty}(t-a) p(t) u(t) d t+\frac{1}{2} \int_{x}^{s}(t-x)(t-a) p(t) u(t) d t
$$

Thus, we can conclude that

$$
u^{\prime}(x) \geq \frac{1}{2} \int_{x}^{\infty}(t-x)^{2} p(t) u(t) d t
$$

on $[a, \infty)$. Furthermore, since

$$
u(x)=u(a)+\int_{a}^{x} u^{\prime}(s) d s,
$$

we have

$$
u(x) \geq u(a)+\frac{1}{2} \int_{a}^{x} \int_{s}^{\infty}(t-s)^{2} p(t) u(t) d t d s
$$

Now, by standard iteration techniques, there is a nonnegative function $w=w(x) d e-$ fined on $[a, \infty)$ such that

$$
w(x)=w(a)+\frac{1}{2} \int_{a}^{x} \int_{s}^{\infty}(t-s)^{2} p(t) w(t) d t d s
$$

Differentiating $w$, we find that

$$
w(x)>0, \quad w^{\prime}(x)>0, \quad w^{\prime \prime}(x)>0, \quad w^{\prime \prime} '(x)<0
$$


and

$$
w^{i v}(x)=-p(x) w(x)
$$

Thus, $w$ is a nonoscillatory solution of (1.2) which implies that all solutions of (1.2) are nonoscillatory (see [3]).

COROLIARY. If $(1.2)$ is oscillatory, then (1.1) is oscillatory.

REMARK. Euler equations can be used to show that the converse of the Theorem does not hold. For example, the equation $y^{i v}-x^{-4} y=0$ is oscillatory while $y^{i v}+x^{-4} y=0$ is nonoscillatory.

We conclude this note with an oscillation criterion for (1.2) and hence for (1.1). This result involves a comparison of (1.2) with a "conditionally" oscillatory second order differential equation.

THiOREM 2. Let $v=v(x)$ be a function such that

$$
\lim _{x \rightarrow \infty} \inf \frac{(x-a)^{2}}{3 ! v(x)} \geq 1
$$

for arbitrary $a \geq 0$ and suppose that the differential equation

$$
z^{\prime \prime}+c v(x) p(x) z=0
$$

is oscillatory for some $c \varepsilon(0,1)$. Then (1.2) is oscillatory.

PROOF. Suppose that $(1.2)$ is nonoscillatury. Then there is a solution $y=y(x)$ of (1.2) satisfying $y(x)>0, y^{\prime}(x)>0, y^{\prime \prime}(x)>0, y^{\prime \prime \prime}(x)>0$ for all $x$ on some half-line $[a, \infty)$. From the Lemma, it follows that

$$
\frac{y(x)}{y^{\prime \prime}(x)} \geq \frac{(x-a)^{2}}{3 !}
$$

on $[a, \infty)$. Note that $(1.2)$ can be written as

$$
z^{\prime \prime}+\frac{p(x) y(x)}{y^{\prime \prime}(x)} z=0, \quad x \geq a,
$$

where $z=y^{\prime \prime}(x)$. Using the Sturm comparison theorem, we conclude that

$$
z^{\prime \prime}+\frac{(x-a)^{2} p(x)}{3 !} z=0
$$

is nonoscillatory. Since $\lim _{x \rightarrow \infty} \inf _{\frac{(x-a)^{2}}{3 ! v(x)}} \geq 1$, corresponding to each $b \in(0,1)$ there exists $t_{b} \geq a$ such that

$$
\frac{(x-a)^{2}}{3 ! v(x)}>b \text { for } x \geq t_{b} \text {. }
$$

Therefore, $b v(x)<\frac{(x-a)^{2}}{3 !}$ on $\left[t_{b}, \infty\right)$ from which it follows that

$$
z^{\prime \prime}+b v(x) p(x) z=0
$$

is nonoscillatory. This contradicts the hypothesis of the theorem and the result is established.

While the techniques in this note are similar to those used in [6], the results are new. 


\section{REFERENCES}

1. Lovelady, D.L. Asymptotic Analysis of Two Fourth Order Linear Differential Equations, Annales Polonici Math. 38 (1980), 109-119.

2. Taylor, W.E., Jr. Oscillation and Asymptotic Behavior in Certain Differential Equations of Odd Order, Rocky Mountain J. Math. 12 (1982), 97-102

3. Leighton, W. and Nehari, Z. On the Oscillation of Solutions of Self-adjoint Linear Differential Equations of the Fourth Order, Trans. Amer. Math. Soc. 89 (1958), 325-377

4. Keener, M.S. On the Solutions of Certain Self-adjoint Differential Equations of Fourth Order, J. Math. Anal. and App1. 33 (1971), 278-304.

5. Ahnad, S. On the Oscillation of Solutions of a Class of Linear Fourth Order Differential Equations, Pacific J. Math. 34 (1970), 289-299.

6. Jones, G.D. An Ordering of Oscillation Types for $y^{(n)}+P y=0$, SIAM J. Math. Ana1. 12 (1981), 72-77.

7. Nehari, Z. Green's Function and Disconjugacy, Archive for Rat. Mech. 62 (1976), 53-76. 


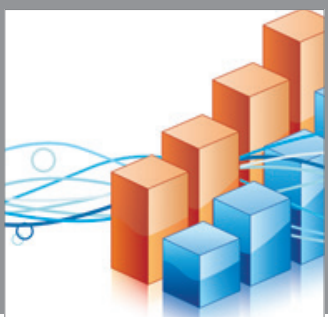

Advances in

Operations Research

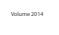

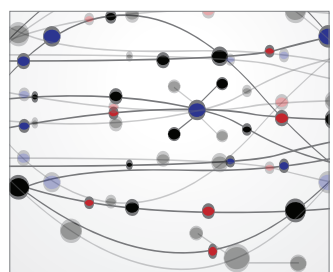

\section{The Scientific} World Journal
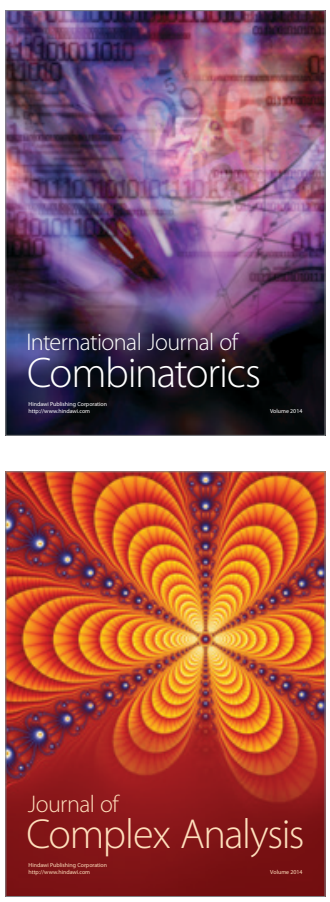

International Journal of

Mathematics and

Mathematical

Sciences
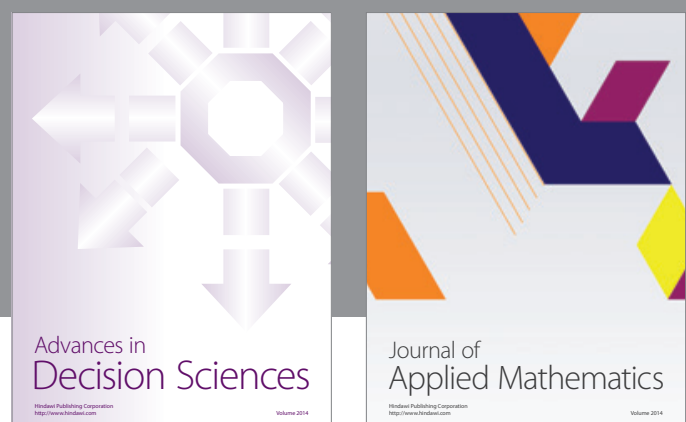

Journal of

Applied Mathematics
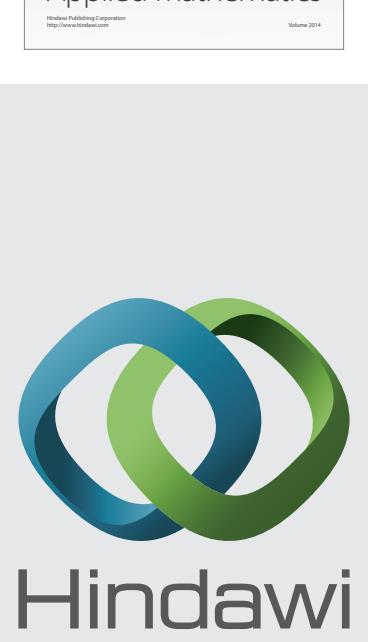

Submit your manuscripts at http://www.hindawi.com
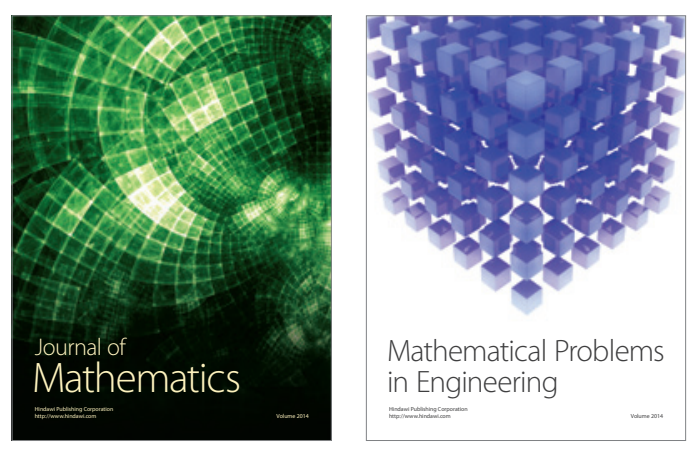

Mathematical Problems in Engineering
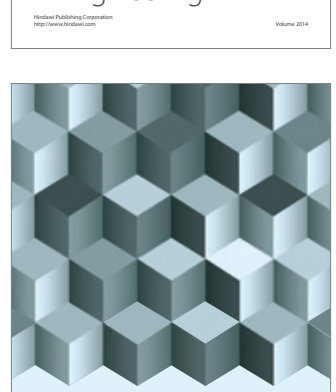

Journal of

Function Spaces
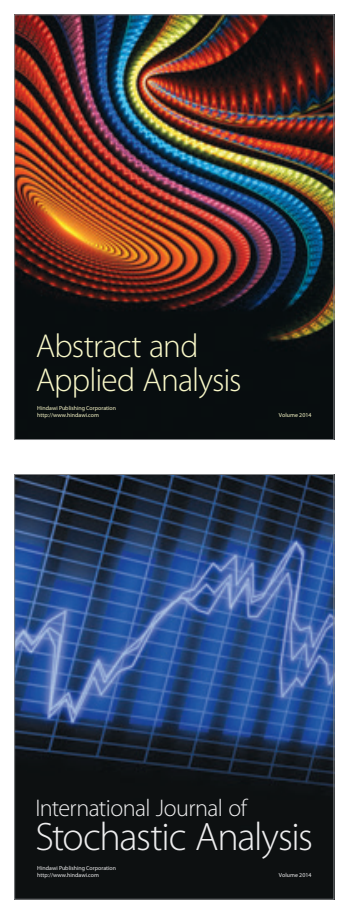

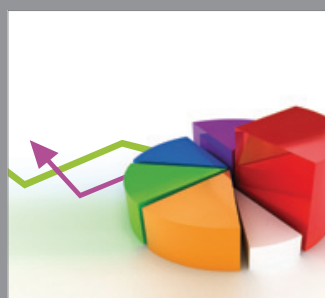

ournal of

Probability and Statistics

Promensencen
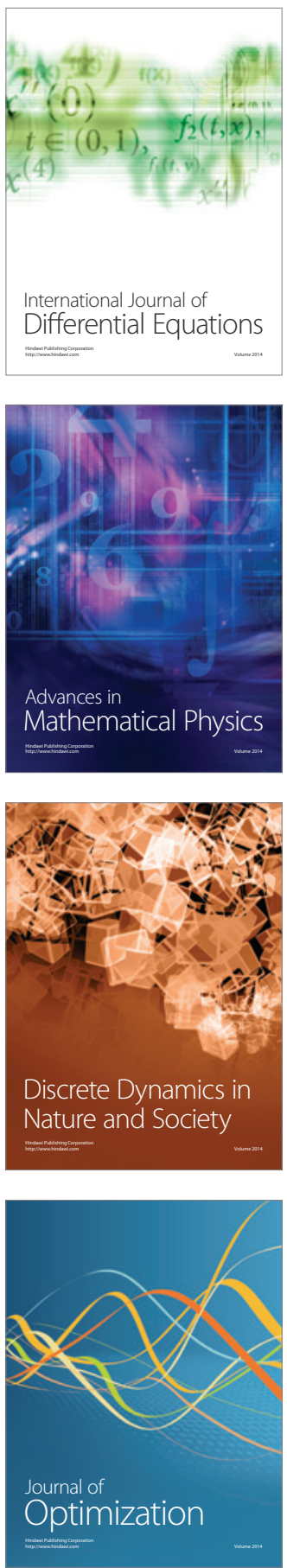